\title{
Wnt-5a regulates proliferation in lung cancer cells
}

\author{
YING HUANG $^{1}$, GUANGXIANG LIU ${ }^{1}$, BO ZHANG ${ }^{2}$, GANG XU $^{2}$, WEI XIONG ${ }^{1}$ and HEPING YANG ${ }^{1}$ \\ Departments of ${ }^{1}$ Respiratory Medicine and ${ }^{2}$ Molecular Genetics, The Third Military Medical University, \\ Chongqing, P.R. China
}

Received March 12, 2009; Accepted June 15, 2009

DOI: 10.3892/or_00000620

\begin{abstract}
Wnt-5a is important in the physiological development and differentiation of lung and is also involved in the regulating proliferation, differentiation and invasion of tumor cells. However, very little is known about the roles of Wnt-5a in the development of lung cancer. The purpose of this study was to explore the role of Wnt-5a in the development of the non-small cell lung cancer, through constructing plasmids containing Wnt-5a sense gene siRNA. We constructed the plasmids containing the Wnt-5a sense gene and siRNA eukaryotic expression vector and transfected it into the human lung squamous carcinoma cell line $\mathrm{H} 157$ and adenocarcinoma cell line A549. Expression of Wnt-5a RNA and protein and proliferation of the cells were measured. Expression of Wnt-5a protein significantly stimulated cell proliferation and transfection with siRNA plasmids suppressed Wnt-5 expression and cell proliferation. The human plasmids containing Wnt-5a sense gene and siRNA eukaryotic expression vector was successfully constructed and transfection to human cancer cells induces cell proliferation. siRNA actively suppressed Wnt-5a expression.
\end{abstract}

\section{Introduction}

The Wnt proteins have been shown to be important regulators in the malignant tumorigenesis (1). Wnt-5a is one of the important members in the large Wnt family and has been demonstrated to play an important role in lung development (2). Absence of Wnt-5a may result in overexpression of fibroblast growth factor-10 (FGF-10), which influences proliferation of the lung epithelium (3).

Wnt-5a has been reported to be predictive of the aggressiveness of gastric cancer (4) and it has also been reported to act as a tumor suppressor (5). Although some

Correspondence to: Dr Ying Huang, Department of Respiratory Medicine, The Third Military Medical University Southwest Hospital, Chongqing, P.R. China

E-mail: liuguoxiang@162.com

Key words: Wnt-5a, gene recombination, RNA interference, cell proliferation, non-small cell lung cancer studies suggest that Wnt-5a is involved in the development of non-small cell lung cancer (NSCLC), the inherent mechanism is still unknown (6-8). Therefore, this study was designed to reconstruct human Wnt-5a gene sense and RNAi by plasmidbased methods in order to determine the role of Wnt-5a expression in NSCLC cell lines in vitro.

\section{Materials and methods}

Construction of pcDNA3.1+-XE58. The Wnt-5a sense gene expression plasmid (pcDNA3.1+-XE58) was constructed by ligating a Wnt-5a cDNA containing the entire coding region from XE58 (Xwnt-5Amyc-CS2+) (provided by R.T. Moon, Washington University, USA) into the NheI and XbaI restriction sites of pcDNA3.1+. The Wnt-5a gene promoter was synthesized as two complementary oligos, with the addition of flanking NheI and $X b a \mathrm{I}$ restriction sequences for cloning purpose. The DNA sequences of the oligos are 5' CTG GCTAGCATGAGAAAGAATCTGTGGACATTTC 3 ' and 5' CTGTCTAGACTACTTGCATGCAAACTGGTCAAC 3'. After the complementary sequences were annealed and digested with NheI and XbaI, the Wnt-5a promoter was directionally inserted into the pcDNA3.1 (+) vector. The plasmid was transformed into $D H 1 O B$ cells and the correct pcDNA3.1+-XE58 plasmid sequence was confirmed by restriction enzyme mapping and DNA sequencing (Fig. 1).

Construction of pAVU6-siWnt-5a. Two siRNA sequences targeting human Wnt-5a (402 and 403) were selected by using GenBank sequence (9). The selection was performed by identifying target sites at arbitrary intervals along the open reading frame of the Wnt-5a gene sequence. As a negative siRNA control, an additional scrambled sequence (401) was produced. The scrambled sequence was not homologous with any known gene in mammalian cells as determined by a Basic Local Alignment Search Tool. The DNA sequence of RNAi 402 was GACCTGGTCTACATCGACC, at bases 1209 to 1228 of the Wnt-5a gene; and the DNA sequence of RNAi 403 was GTGCAAGAAGTGCACGGAG, at bases 1415 to 1434 of the Wnt-5a gene. Three DNA sequences were chemically synthesized as oligos flanked with SalI at the 5' end and XbaI at $3^{\prime}$ end for cloning purpose (Table I). The vector map of the pAVU6-siWnt-5a is shown in Fig. 2.

Construction of plasmids. The plasmids were constructed according to previously reported methods $(10,11)$. The plasmid 
Table I. Two chemically synthesized DNA sequences (402 and 403) and control sequence (401).

402:

403:

401:
5' TCGACGACCTGGTCTACATCGACCTCTGGGTCGATGTAGACCAGGTCTTTTTGGT

5' CTAGACCAAAAAGACCTGGTCTACATCGACCCAGAGGTCGATGTAGACCAGGTCG

5' TCGACGTGCAAGAAGTGCACGGAGTCTGCTCCGTGCACTTCTTGCACTTTTTGGT

5' CTAGACCAAAAAGTGCAAGAAGTGCACGGAGCAGACTCCGTGCACTTCTTGCACG

5' TCGACGGTGGAACTGCATGACTGTTCTGACAGTGCTGCAGTTCCACCTTTTTGGT

5' CTAGACCAAAAAGGTGGAAGCACAGCACTGTCAGAACAGTGCTGCAGTTCCACCG

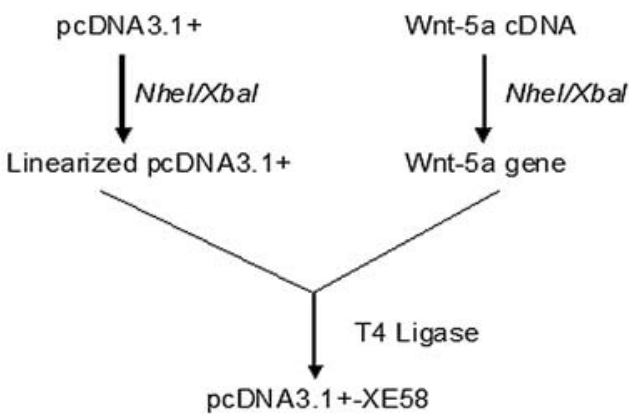

Figure 1. Schematic view of pcDNA3.1+-XE58 construction

pAVU6+27 was isolated using previously reported methods (13). The digestion product was annealed with the RNAi sequences at $37^{\circ} \mathrm{C}$ for 60 min according to published methods $(10,12,13)$. The recovery and purification of the plasmids were performed according to the manufacturer's instruction of kit (Omega, USA). The linearized plasmid was ligated and transformed to $D H 10 B(14,15)$. The competent cells were prepared by the method reported previously (16).

Transfection of plasmids and stable clone selection. Human lung squamous carcinoma cell line H157 and human lung adenocarcinoma cell line A549 were seeded in 24-well plates at $5 \times 10^{4}$ cells/well in culture media without antibiotics $24 \mathrm{~h}$ prior to transfection. Both $\mathrm{H} 157$ and A549 cells at $85-90 \%$ confluence were transfected using lipofectamine 2000 according to the manufacturer's instructions (Invitrogen, USA). Stably expressing Wnt-5a was generated in both cell lines and the cell clones were subcultured and maintained at the lowest lethal concentration of G418 for 18 to 21 days.

H157 and A549 cells transfected with pAVU6-siWnt-5a and pcDNA3.1+-XE58 (H157-siWnt-5a, A549-siWnt-5a, H157-XE58 and A549-XE58) were used as study cells; cells transfected with pAVU6_27 and pcDNA3.1+ were positive controls (H157-pAVU6, H157-pcDNA3.1+, A549-pAVU6 and A549-pcDNA3.1+) and cells without transfection were negative controls (H157 and A549).

Semiquantitative RT-PCR analysis. Total RNA was isolated from both $\mathrm{H} 157$ and A549 cells according to the method described previously $(17,18)$. To quantitate the RNA results, image analysis was performed on Image Analysis System (Eppendorf, Germany). The mean band intensity of triplicate experimental samples was determined and compared to the band intensity of the empty vector-transfected control cells.

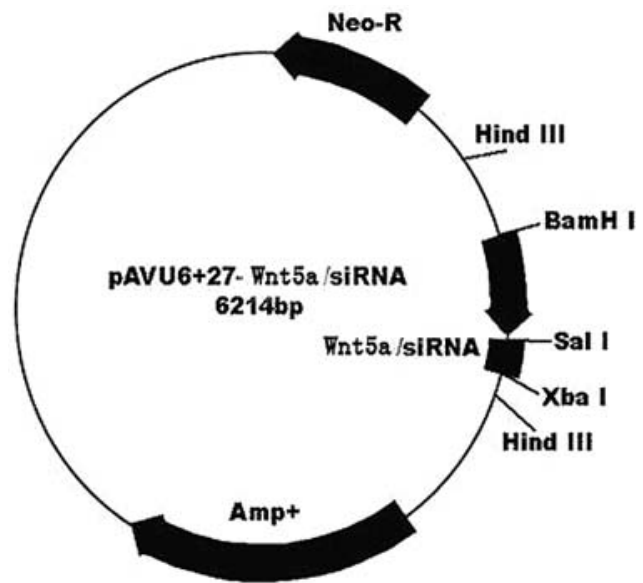

Figure 2. Vector map of pAVU6-siWnt-5a plasmid. pAVU6-siWnt-5a was digested with BamHI and HindIII, the size of product was 400 base pairs.

Western blot analysis. The relative Wnt-5a protein lysis was measured by observation of the gray scale, according to the method reported previously $(17,18)$. Wnt-5a proteins were detected by electrochemiluminescence protein detection system (Gel Dco, USA).

Cell proliferation. Cellular proliferation was determined by measuring the total number of viable cells at $0,24,48$ and $72 \mathrm{~h}$ after plating cells at $1 \times 10^{3} /$ well in 96-wells with $200 \mu 1$ in each well. The total number of cells at each time point was determined by methyl thiazolyl tetrazolium assay. The absorbance (A) of cells was measured at $490 \mathrm{~nm}$ using the Ultra Microplate Reader (Dynex, USA). Cell numbers were calculated from a standard curve relating cell number to A values (OD) at $490 \mathrm{~nm}$.

Statistical analysis. All experimental data were analyzed using a statistical software package SPSS10.0. T-test was used and data were demonstrated as mean $\pm \mathrm{SD}$. $\mathrm{P}<0.05$ was considered statistically significant.

\section{Results}

The plasmid of pAVU6-siWnt-5a was digested with BamHI and HindIII, which demonstrated that the product of 400 base pairs in length was visualized on $0.8 \%$ gel, indicating that pAVU6-siWnt-5a was successfully reconstructed (Fig. 3A). The plasmid of pcDNA3.1+-XE58 was digested using BamHI, which showed that two bands of products of 1200 base pairs were obtained whereas the product of 5400 base pairs was 
A

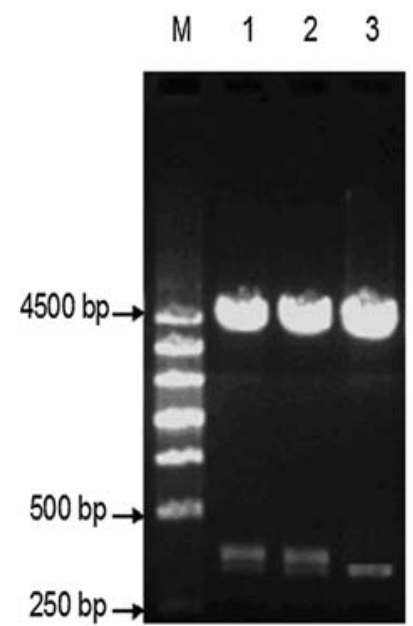

B

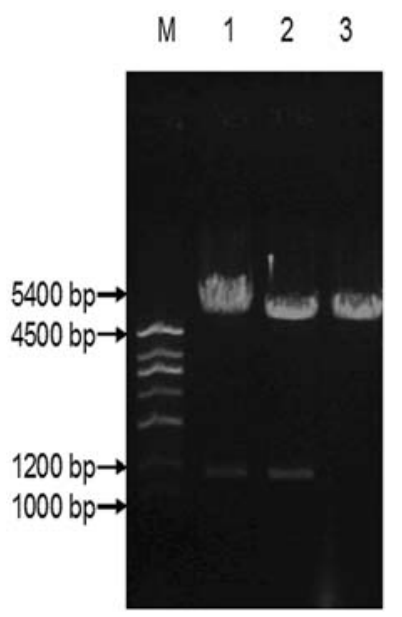

Figure 3. (A) Plasmid of pAVU6-siWnt-5a digested with BamHI/HindIII. M, marker; 1 and 2, pAVU6-siWnt-5a digested with BamHI/HindIII. 3, pAVU6+27 digested with BamHI/HindIII. (B) Plasmid of pcDNA3.1+-XE58 digested with BamHI. M, marker; 1 and 2, pcDNA3.1+-XE58 digested with BamHI; 3, pcDNA3.1+ digested with BamHI.

Table II. Effects of Wnt-5a mRNA expression among groups.

\begin{tabular}{lcc}
\hline Cells & Wnt-5a mRNA & P-value \\
\hline H157 & $0.45 \pm 0.03$ & $\mathrm{P}>0.05$ \\
H157-pcDNA3.1+ & $0.45 \pm 0.01$ & $\mathrm{P}>0.05$ \\
H157-pAVU6 & $0.44 \pm 0.05$ & $\mathrm{P}<0.05^{\mathrm{a}}$ \\
H157-XE58 & $0.89 \pm 0.08$ & $\mathrm{P}<0.05^{\mathrm{b}}$ \\
H157-siWnt-5a & $0.23 \pm 0.06$ & $\mathrm{P}<0.05^{\mathrm{c}}$ \\
A549 & $0.38 \pm 0.04$ & $\mathrm{P}>0.05$ \\
A549- pcDNA3.1+ & $0.43 \pm 0.01$ & $\mathrm{P}>0.05$ \\
A549-pAVU6 & $0.42 \pm 0.09$ & $\mathrm{P}<0.05^{\mathrm{a}}$ \\
A549-XE58 & $0.77 \pm 0.07$ & $\mathrm{P}<0.05^{\mathrm{b}}$ \\
A549-siWnt-5a & $0.19 \pm 0.03$ & $\mathrm{P}<0.05^{\mathrm{c}}$ \\
\hline
\end{tabular}

${ }^{\mathrm{a} C e l l s ~ t r a n s f e c t e d ~ w i t h ~ p c D N A 3.1+-X E 58 ~ v s . ~ c o n t r o l ~ g r o u p . ~}{ }^{\mathrm{b}} \mathrm{Cells}$ transfected with pAVU6-siWnt-5a vs. control group. ' $\mathrm{pcDNA3.1+-}$ XE58-transfected cells vs. pAVU6-siWnt-5a-transfected cells.

in the empty vector (pcDNA3.1+), indicating that pcDNA3.1+ -XE58 was successfully reconstructed (Fig. 3B).

The expression of Wnt-5a mRNA in H157 and A549 cells transfected with pcDNA3.1+-XE58 was significantly increased, compared to Wnt-5a mRNA expression in the empty vector-transfected control cells. Cells transfected with pAVU6-siWnt-5a showed clearly decreased expression of Wnt-5a mRNA, suggesting that the siRNA plasmid significantly reduced expression of Wnt-5a mRNA (Table II). Similarly, the expression of Wnt-5a protein in H157 and A549 cells transfected with pcDNA3.1+-XE58 was significantly increased compared to the empty vector-transfected control cells. Cells transfected with pAVU6-siWnt-5a showed clearly decreased expression of Wnt-5a protein, suggesting that the siRNA plasmid significantly reduced expression of Wnt-5a mRNA (Table III). The findings of the expression of Wnt-5a protein and mRNA indicate that Wnt-5a can be upregulated and downregulated in lung cancer cells.
Table III. Effects of Wnt-5a proteins among groups.

\begin{tabular}{lcc}
\hline Cells & Wnt-5a protein & P-value \\
\hline H157 & $7.71 \pm 3.84$ & $\mathrm{P}>0.05$ \\
H157-pcDNA3.1+ & $7.73 \pm 3.35$ & $\mathrm{P}>0.05$ \\
H157-pAVU6 & $7.68 \pm 3.29$ & $\mathrm{P}<0.05^{\mathrm{a}}$ \\
H157-XE58 & $14.5 \pm 2.86$ & $\mathrm{P}<0.05^{\mathrm{b}}$ \\
H157-siWnt5a & $4.02 \pm 0.84$ & $\mathrm{P}<0.05^{\mathrm{c}}$ \\
A549 & $7.23 \pm 2.97$ & $\mathrm{P}>0.05$ \\
A549- pcDNA3.1+ & $7.57 \pm 2.02$ & $\mathrm{P}>0.05$ \\
A549-pAVU6 & $7.44 \pm 2.31$ & $\mathrm{P}<0.05^{\mathrm{a}}$ \\
A549-XE58 & $13.6 \pm 3.48$ & $\mathrm{P}<0.05^{\mathrm{b}}$ \\
A549-siWnt-5a & $3.29 \pm 3.17$ & $\mathrm{P}<0.05^{\mathrm{c}}$ \\
\hline
\end{tabular}

${ }^{a}$ Cells transfected with pcDNA3.1+-XE58 vs. control group. ${ }^{\mathrm{b}}$ Cells transfected with pAVU6-siWnt-5a vs. control group. 'pcDNA3.1+XE58-transfected cells vs. pAVU6-siWnt-5a-transfected cells.

The proliferation trends was significantly increased in both cell lines transfected with pcDNA3.1+-XE58 (H157-XE58 and A549-XE58), compared to cells transfected with empty vector and the untransfected cells. The overexpression of Wnt-5a induced significantly increased proliferation at all time-points of 24, 48 and $72 \mathrm{~h}$. Transfection of the plasmid pAVU6-siWnt-5a (H157-siWnt-5a and A549-siWnt-5a cells) suppressed the expression of Wnt-5a. Expression of Wnt-5a correlates with increased cellular proliferation in NSCLC, suggesting that Wnt-5a may regulate proliferation in NSCLCderived cells (Figs. 4 and 5).

\section{Discussion}

In 1998, the term RNA interference (RNAi) was coined referring to the phenomenon of post-translational silencing of gene expression after the introduction of specific doublestranded RNA (dsRNA) fragments of 21-23 nucleotides, known as small interfering RNA (siRNA) $(19,20)$. RNAi has 


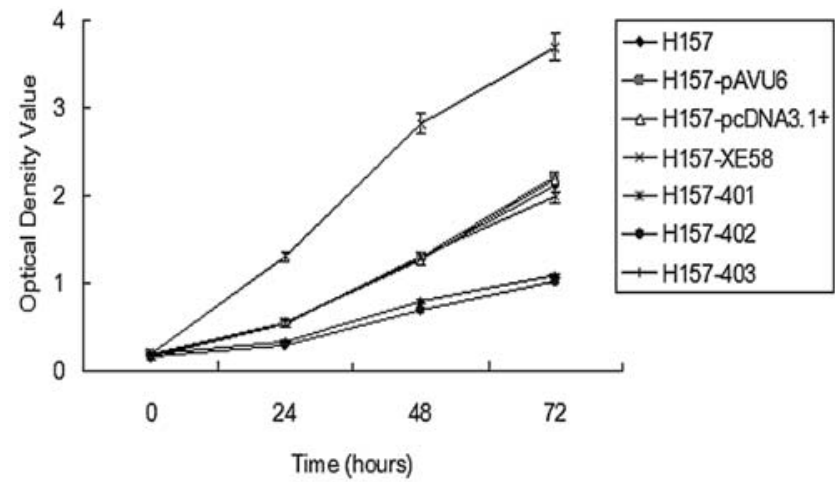

Figure 4. Trends of cell growth of $\mathrm{H} 157$ transfected with pAVU6-siWnt-5a and pcDNA3.1+-XE58. The growth of H157 cell line transfected with pcDNA3.1+-XE58 (H157-XE58) was significantly increased compared to cells transfected with empty vector and untransfected cells.

emerged as an important experimental mechanism for silencing gene expression. This phenomenon can result in highly specific suppression of gene expression. RNAi promises not only to provide a reliable and rapid approach to the analysis of gene function, but also to revolutionize key areas of gene therapy, especially against neoplastic diseases (21). RNAi technology has been employed as gene therapy against different malignances, but so far Wnt-5a RNAi has not been documented as a potential gene therapy against lung cancer. To our knowledge, the present study may be the first constructing human Wnt-5a sense gene and RNAi eukaryotic expression vector.

It is well known that efficient and stable gene expression is crucial for the study of gene functions. Upregulation of gene expression is produced by the transfection of Wnt-5a sense gene, while downregulation through RNA interference is completed by two means: i) shRNA transcribed from two RNA polymerase-dependent U6 and H1 promoters is cleaved by Dicer to produce 21-23 nucleotide dsRNA fragments with two nucleotide 3 ' end overhangs, i.e. siRNAs and ii) the 5 ' sense and $5^{\prime}$ antisense strands, individually transcribed from two RNA polymerase-dependent U6 and $\mathrm{H} 1$ promoters in different plasmids, form internal hairpin-like structures. The shRNA approach can significantly silence its target gene expression in cancer cells (22). This approach is suitable for the long-term study of RNAi due to its high transfection efficiency and stable expression after transfection. For the construction of the plasmid pAVU6-siWnt-5a to downregulate Wnt-5a expression, plasmid pAVU6+27 was used. This plasmid contains the RNA polymerase-dependent U6 snRNA gene promoter, which accurately initiates and ends transcription at specific sequences. These constructions produce small inverted repeats, separated by a spacer of three to nine nucleotides, termed short hairpin RNAs (shRNAs), which are processed by Dicer into siRNAs. In addition to a defined initiation sequence, the U6 polymerase-III promoter terminates with TTTT or TTTTT. The products are shRNAs that contain a series of uridines at the 3 ' end, a feature that seems to favor RNAi (21).

Wnt-5a sense gene plasmid (pcDNA3.1+-XE58) was constructed by inserting a Wnt-5a cDNA containing the entire coding region from XE58 (Xwnt-5Amyc-CS2+) into the NheI/XbaI sites of pcDNA3.1+. The Wnt-5a gene was

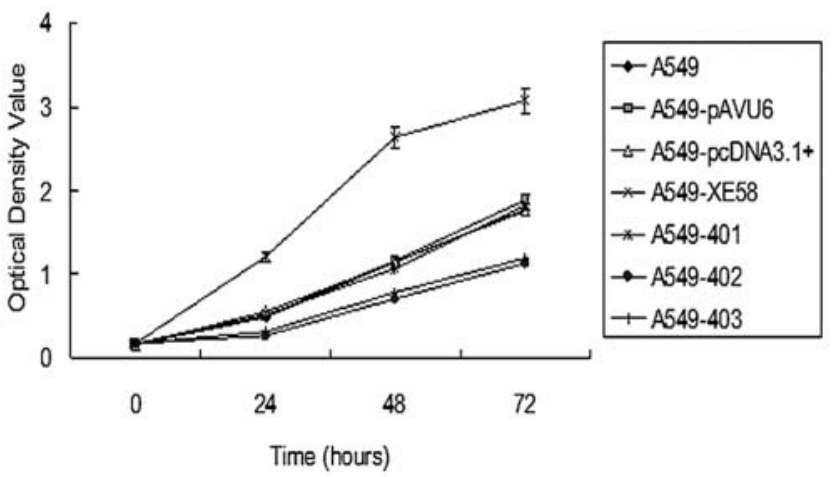

Figure 5. Trends of cell growth of A549 transfected with pAVU6-siWnt-5a and pcDNA3.1+-XE58. The growth of A549 cell line transfected with pcDNA3.1+-XE58 (A549-XE58) was significantly increased compared to cells transfected with empty vector and untransfected cells.

confirmed and showed that the recombinant was successfully obtained.

Expression levels of Wnt-5a mRNA and protein in H157 and A549 cells were analyzed. The empty vector-transfected cells and untransfected control cells showed no significant difference in expression levels of Wnt-5a mRNA and protein. Significantly elevated expression of Wnt-5a mRNA and protein were seen in both H157-XE58 and A549-XE58, compared to empty vector-transfected cell group and control group, suggesting that Wnt-5a mRNA and protein are upregulated in the H157 and A549 cells transfected with recombinant pcDNA3.1+-XE58 containing Wnt-5a sense gene. Cells transfected with pAVU6-siWnt-5a showed clearly a decrease in expression of Wnt-5a mRNA and protein, compared to the empty vector-transfected clones and control clones, suggesting that the siRNA plasmid was specific for suppressing the expression of target gene. These results suggest that the designation of siRNA is characterized by the target specificity and is reached to the effect of suppression of target gene.

Thus, to the best of our knowledge, this is the first study that the recombinant pcDNA3.1+-XE58 and pAVU6-siWnt5 a were successfully constructed and transfected into the human lung squamous carcinoma cell line H157 and adenocarcinoma cell line A549, yielding stable transfectants, which provide with a fundamental access to further research into the function of Wnt-5a.

One of the characteristics of malignant cancer is uncontrolled cell growth. Infinite proliferation as an essential characteristic of cancer cells is the precondition for its invasion and metastasis. Our results indicate that the expression of Wnt-5a correlates with cellilar proliferation in NSCLC, and that the high expression of Wnt-5a significantly enhanced the cell proliferation, suggesting that Wnt-5a regulate proliferation in NSCLC-derived cells. These findings are consistent with those previously reported (15), which demonstrated that overexpression of the Wnt-5a gene directly enhanced the motility and invasion of metastatic melanoma.

In conclusion, plasmids containing human Wnt-5a sense gene and siRNA were successfully constructed and stable transfectants were obtained in the present study. The findings indicate that the upexpression of Wnt-5a enhanced proliferation 
of NSCLC, while downregulation of Wnt-5a decreased proliferation, suggesting that Wnt-5a is involved in the development of NSCLC. Therefore, Wnt-5a gene plays an important role in the development of NSCLC and may act as a target for NSCLC gene therapy.

\section{Acknowledgements}

We greatly appreciate Professor D.R. Engelke from University of Michigan for providing the plasmid pAVU6+27, Professor Randall Moon from University of Washington for providing XE58, Professor Zhengtang Chen and Dr Baoguo Kang from Third Military Medical University, China, for providing cell lines H157 and A549.

\section{References}

1. Lange C, Mix E, Rateitschak K and Rolfs A: Wnt signal pathways and neural stem cell differentiation. Neurodegener Dis 3: 76-86, 2006.

2. Masckauchan TN, Agalliu D, Vorontchikhina M, et al: Wnt5a signaling induces proliferation and survival of endothelial cells in vitro and expression of MMP-1 and Tie-2. Mol Biol Cell 17: 5163-5172, 2006.

3. Iozzo RV, Eichstetter I and Danielson KG: Aberrant expression of the growth factor Wnt-5a in human malignancy. Cancer Res 55: 3495-3499, 1995.

4. Kurayoshi M, Oue N, Yamamoto H, et al: Expression of Wnt-5a is correlated with aggressiveness of gastric cancer by stimulating cell migration and invasion. Cancer Res 66: 10439 10448, 2006.

5. Dejmek J, Dejmek A, Safholm A, et al: Wnt-5a protein expression in primary dukes $\mathrm{B}$ colon cancers identifies a subgroup of patients with good prognosis. Cancer Res 65: 9142 $9146,2005$.

6. Taki M, Kamata N, Yokoyama K, et al: Down-regulation of Wnt-4 and up-regulation of Wnt-5a expression by epithelialmesenchymal transition in human squamous carcinoma cells. Cancer Sci 94: 593-597, 2003.

7. Huang CL, Liu D, Nakano J, et al: Wnt5a expression is associated with the tumor proliferation and the stromal vascular endothelial growth factor - an expression in non-small cell lung cancer. J Clin Oncol 23: 8765-8773, 2005.
8. Ungar AR, Kelly GM and Moon RT: Wnt4 affects morphogenesis when misexpressed in the zebrafish embryo. Mech Dev 52: 153-164, 1995.

9. Strathdee CA, McLeod MR and Hall JR: Efficient control of tetracycline responsive gene expression from an autoregulated bi-directional expression vector. Gene 229: 21-29, 1999.

10. Good PD, Krikos AJ, Li SX, et al: Expression of small; therapeutic RNAs in human cell nuclei. Gene Ther 4: 45-54, 1997.

11. Paul CP, Good PD, Winer I, et al: Effective expression of small interfering RNA in human cells. Nat Biotechnol 20: 505-508, 2002.

12. Hammond SM, Caudy AA and Hannon GJ: Post-transcriptional gene silencing by double-stranded RNA. Nat Rev Genet 2: 110-119, 2001 .

13 Tillett D and Neilan BA: An improved method for the purification of large DNA Fragments from agarose gels using WizardPlusSV Columns. Anal Biochem 269: 218-219, 1999.

14. Cohen SN, Chang ACY, Boyer HW, et al: Construction of biologically functional bacterial plasmids in vitro. Proc Natl Acad Sci USA 73: 3240-3244, 1973.

15. Hanahan D: Studies on transformation of Escherichia coli with plasmids. J Mol Biol 16: 557-580, 1983.

16. Comelis S, Vandenbranden M and Ruysschaert JM: Role of intracellular cationic liposome-DNA complex dissociation in transfection mediated by cationic lipids. DNA Cell Biol 21: 91-97, 2002.

17. Dalby B, Cates S, Harris A, et al: Advanced transfection with lipofectamine 2000 reagent: primary neurons, siRNA, and highthroughput applications. Methods 33: 95-103, 2004.

18. Ullrich A, Shine J, Chirgwin J, et al: Rat insulin genes: Construction of plasmids containing Te coding sequences. Science 196: 1313-1319, 1977.

19. Chomczynski P and Sacchi N: Single-step method of RNA isolation by acid guanidinium thiocyanate-phenol-chloroform extraction. Anal Biochem 162: 156-159, 1987.

20. Filipowicz W, Jaskiewicz L, Kolb FA, et al: Post-transcriptional gene silencing by siRNAs and miRNAs. Curr Opin Struct Biol 15: 331-341, 2005.

21. Weeraratna AT, Jiang Y, Hostetter G, et al: Wnt5a signaling directly affects cell motility and invasion of metastatic melanoma. Cancer Cell 1: 279-288, 2002.

22. Lipardi C, Wei Q and Paterson BM: RNAi as random degradative PCR: siRNA primers convert mRNA into dsRNAs that are degraded to generate new siRNAs. Cell 107: 297-307, 2001. 